Cahiers d'études italiennes

1 | 2004

NOVECENTO... E DINTORNI

Dire la guerre?

\title{
Luoghi comuni su Berlusconi
}

\section{Valerio Evangelisti}

\section{OpenEdition}

\section{Journals}

Edizione digitale

URL: http://journals.openedition.org/cei/969

DOI: $10.4000 /$ cei.969

ISSN: 2260-779X

\section{Editore}

UGA Éditions/Université Grenoble Alpes

\section{Edizione cartacea}

Data di pubblicazione: 15 novembre 2004

Paginazione: 226-234

ISBN: 978-2-84310-057-4

ISSN: 1770-9571

Notizia bibliografica digitale

Valerio Evangelisti, «Luoghi comuni su Berlusconi», Cahiers d'études italiennes [Online], 1 | 2004, online dal 15 mai 2006, consultato il 26 mars 2021. URL: http://journals.openedition.org/cei/969 ; DOI: https://doi.org/10.4000/cei.969 


\title{
LUOGHI COMUNI SU BERLUSCONI
}

\author{
Valerio Evangelisti
}

Ho l'impressione che insistere troppo sugli aspetti pagliacceschi di Silvio Berlusconi, come fa gran parte della stampa internazionale, distragga dalla sostanza della sua azione di governo. Non vi è dubbio che si tratti di un personaggio dalla volgarità innata, simile per atteggiamenti e per cultura a certi commessi viaggiatori di vecchio stampo. Non vi è dubbio, altresì, che buona parte della sua attività sia volta a stravolgere le istituzioni in senso autocratico, oppure a porlo al riparo dai guai giudiziari che lo sovrastano. Tuttavia è bene mettere in chiaro alcune cose. Non è un fascista. Non è un mafioso. Non è un Pulcinella (questo ruolo lo attribuirei piuttosto a una gran parte dei collaboratori che si è scelto, capaci di lasciare increduli per la loro stupidità). È, se vogliamo, molto peggio di tutto ciò.

Silvio Berlusconi rappresenta, ai miei occhi, una variante tutta italiana di ciò che fu Margaret Thatcher per l'Inghilterra. Ricordiamocelo. Una dama estranea al tradizionale tessuto conservatore inglese travolge la leadership del partito, si fa portavoce di ceti medi giunti a una loro coscienza di classe, si pone in sintonia con Ronald Reagan e vara un programma ultraliberista indirizzato a scalzare Welfare State e sindacati. La trasformazione è talmente radicale da sopravvivere alla scadenza del mandato. Tony Blair raccoglie di fatto, lo voglia o no, l'eredità anti-keynesiana della Thatcher, e tutto l'Occidente è tuttora improntato alla visione del mondo propria dell'asse Thatcher-Reagan: ciò che Ignacio Ramonet ha felicemente definito il "pensiero unico".

Il percorso italiano è simile, anche se invertito nelle apparenze ideologiche. Spetta a un socialista, Bettino Craxi, prendere atto della raggiunta consapevolezza di classe dei ceti medi. L'innesco è, nel 1980, una marcia di 40.000 quadri della Fiat contro i sindacati che hanno occupato la sede di Mirafiori dell'azienda. Pochi intuiscono, a quel tempo, che si tratta di una svolta epocale, ma Craxi sì. Mette mano all'indebolimento di un Welfare State che pareva intangibile, va allo scontro frontale con le organizzazioni operaie. Parallelamente, incoraggia in tutti i modi l'arricchimento delle classi medie fino a quel momento vittime di un'identità incerta. Professionisti, imprenditori di medio calibro, ceti impiegatizi del settore privato, operatori nel campo finanziario o commerciale, sono 


\title{
LIEUX COMMUNS SUR BERLUSCONI
}

\author{
Valerio Evangelisti
}

Traduction française de Christophe Mileschi, revue par l'auteur

J'ai l'impression qu'à trop insister sur les côtés clownesques de Silvio Berlusconi, comme le fait une bonne partie de la presse internationale, on risque de perdre de vue la substance de son action de gouvernement. Il ne fait pas de doute qu'il s'agit d'un personnage naturellement vulgaire, qui fait penser, par ses attitudes et par sa culture, à certains commis voyageurs du temps jadis. Il ne fait pas de doute non plus qu'une large part de son activité vise à remodeler les institutions dans un sens autocratique, ou bien à lui permettre de se mettre à l'abri des ennuis judiciaires qui pèsent sur lui. Il est bon, cependant, de clarifier certaines choses. Berlusconi n'est pas un fasciste. Ce n'est pas un mafieux. Ce n'est pas un Polichinelle (rôle que j'attribuerais plus volontiers à bon nombre des collaborateurs qu'il s'est choisis, dont la bêtise est parfois telle qu'elle peut laisser incrédule). C'est, si l'on veut, bien pire que tout cela.

Silvio Berlusconi représente à mes yeux une variante typiquement italienne de ce que fut Margaret Thatcher pour l'Angleterre. Rappelonsnous. Une dame étrangère au monde conservateur anglais traditionnel met sens dessus dessous le leadership de son parti, devient porte-parole des classes moyennes qui ont atteint à leur façon une conscience de classe, se met en phase avec Ronald Reagan, et lance un programme ultralibéral visant à démolir la protection sociale et les syndicats. La transformation est tellement radicale qu'elle survit à la fin du mandat. De fait, Tony Blair recueille, qu'il le veuille ou non, l'héritage anti-keynésien de Thatcher, et aujourd'hui encore, c'est l'Occident tout entier qui reste marqué par la vision du monde propre à l'axe Thatcher-Reagan : ce qu'Ignacio Ramonet a joliment appelé «la pensée unique».

Le parcours italien est semblable, bien qu'il aille en sens inverse, si l'on s'en tient aux apparences idéologiques. C'est à un socialiste, Bettino Craxi, qu'il revient de prendre acte de la conscience de classe qu'ont acquise les classes moyennes. Le déclic se produit en 1980, avec la marche de 40000 cadres de Fiat contre les syndicats qui ont occupé le siège de l'entreprise à Mirafiori. Peu nombreux sont ceux qui, à l'époque, devinent qu'il s'agit là 
incentivati ad arricchirsi senza freni, e a prendere atto del potere raggiunto.

Silvio Berlusconi è, di Bettino Craxi, il diretto erede. Con un passaggio intermedio, però. Durante i governi che si succedono dopo l'esilio di Craxi (giudicato in tribunale colpevole di essere al centro di un esteso sistema di corruzione), gran parte della sinistra abbraccia anch'essa la causa dei ceti medi arricchiti, e modella sulle loro istanze il proprio programma. Questo è naturalmente anti-keynesiano, centrato su una priorità di risanamento del debito pubblico di chiara impronta monetarista, favorevole alla privatizzazione dei settori dell'economia ancora in mani statali, ostile alla stabilità del lavoro e all'erogazione gratuita dei servizi, incline ad agevolare l'azionariato quale sistema di risparmio e i fondi di investimento quali succedanei alle pensioni di Stato.

Niente più egualitarismo, niente più tentativi di allargare dal basso gli spazi democratici. La sinistra ridisegnata procede anzi a una limitazione sensibile della democrazia. Il sistema politico, da proporzionale che era, si fa maggioritario : nessun cittadino troverà più in parlamento una forza che lo rappresenti per intero, e il potere diverrà autonomo rispetto alla società e alle sue tensioni. Più o meno la visione di Craxi.

Con una simile scelta, la sinistra - fusa in una coalizione con alcuni partiti di centro dalle molte contraddizioni interne - spera forse di perpetuare il proprio comando. Invece offre a Berlusconi il modo per fruire in parlamento di una sovrarappresentazione del suo partito, Forza Italia, e gli regala i numeri per un uso "sovversivo" del potere governativo, capace di intaccare la stessa Costituzione.

Nel definire le caratteristiche dell'ascesa di Silvio Berlusconi alla presidenza del Consiglio e a poteri amplissimi, occorrerebbe astenersi dal sopravvalutare il suo monopolio sui mezzi di comunicazione di massa, per quanto indubbio. Tale monopolio, di cui Craxi incoraggiò la formazione, avrebbe a mio parere un impatto circoscritto se non si sommasse a una composizione sociale profondamente alterata. La volgarità della programmazione delle reti televisive di proprietà di Berlusconi (e oggi anche delle reti pubbliche cadute in sue mani) è fuor di discussione, ma trova rispondenza nella volgarità culturale propria dei ceti medi giunti a "coscienza di classe».

La parola d'ordine «arricchitevi!» non poteva che creare legioni di parvenus, edonisti in privato e privi, a livello sociale, di qualsiasi etica - proprio come era avvenuto in Inghilterra nel periodo thatcheriano. Rispetto 
d'un tournant historique. Mais Craxi, oui. Il met la première main à l'affaiblissement d'un Welfare State qui paraissait intouchable, il va au heurt frontal avec les organisations ouvrières. Parallèlement, il encourage de toutes les façons possibles l'enrichissement des classes moyennes, jusque là aux prises avec une identité incertaine. Professions libérales, entrepreneurs de moyen calibre, employés du secteur privé, opérateurs du champ de la finance ou du commerce sont incités à s'enrichir sans retenue, et à prendre la mesure du pouvoir qu'ils détiennent.

Silvio Berlusconi est l'héritier direct de Bettino Craxi. Mais avec une étape intermédiaire. Pendant les gouvernements qui se succèdent après l'exil de Craxi (que les tribunaux ont jugé coupable d'avoir été au centre d'un vaste système de corruption), une partie importante de la gauche embrasse à son tour la cause des classes moyennes enrichies, et calque son propre programme sur leurs priorités. C'est, bien évidemment, un virage anti-keynésien, qui met l'accent sur une priorité - l'assainissement de la dette publique - dont la matrice est clairement monétariste, qui est favorable à la privatisation des secteurs de l'économie encore contrôlés par l'État, hostile à la stabilité du travail et à la gratuité des services, et qui tend à encourager l'actionnariat comme système d'épargne et les fonds de pension comme succédanés du système public des retraites.

Fini l'égalitarisme, finies les tentatives pour élargir par le bas les espaces démocratiques. La gauche redessinée met au contraire en œuvre une limitation sensible de la démocratie. Le système politique, de proportionnel qu'il était, devient majoritaire: aucun citoyen ne trouvera plus au parlement une force qui le représente réellement, et le pouvoir prendra son autonomie par rapport à la société et à ses tensions. Voici, plus ou moins, la vision de Craxi.

En faisant de tels choix, la gauche - qui se fond dans une coalition avec certains partis centristes dont les contradictions internes sont nombreuses - espère peut-être faire durer son règne. C'est tout l'inverse: elle offre à Berlusconi la possibilité de tirer profit, au parlement, d'une surreprésentation de son parti, Forza Italia, et lui offre les clefs d'une utilisation «subversive» du pouvoir de gouvernement, en mesure d'entamer la Constitution elle-même.

S'agissant de définir les caractéristiques de l'ascension de Silvio Berlusconi à la présidence du Conseil et à des pouvoirs très étendus, il conviendrait de s'abstenir de surévaluer, encore qu'il soit incontestable, le monopole qu'il exerce sur des moyens de communication de masse. Ce monopole, dont Craxi encouragea l'apparition, n'aurait à mon avis qu'un 
al conservatorismo tradizionale, manca loro non solo la dignità aristocratica, ma anche l'intelaiatura morale legata al mondo della produzione, sia pure visto da un'angolatura padronale. Le vecchie virtù capitalistiche del risparmio, della moderazione, del reinvestimento saggio e prudente sono spazzate via.

Del resto, quale rapporto diretto con la produzione possono avere stuoli di avvocati, di notai, di assicuratori, di operatori dell'informatica, della comunicazione e dello spettacolo, di imprenditori dell'edilizia, di giocatori di borsa usi a far soldi con i soldi? Quale etica può nascere da tutto questo? E se si tratta di quadri o manager legati all'universo produttivo, la facile prospettiva di ricavare denaro dalla manodopera sottocosto dei paesi dell'Est, o di spostare l'aziendina in qualche regione povera del mondo resa accessibile dalla mondializzazione, basta a distoglierli dall'antica fandonia liberale per cui la ricchezza di una minoranza può tradursi in ricchezza sociale, per intrinseci meccanismi a cascata.

Non sono dei fascisti, costoro: sarebbe troppo impegnativo. Sono invece semplicemente dei cinici. Non cercano alibi alle loro azioni : il più forte e fortunato vince, questa è l'unica regola. Chi perde si arrangi, la società non è fatta per lui. E se ci sono leggi ispirate a concezioni moralistiche obsolete, basta ignorarle o cancellarle. Sono i rapporti di forza che fondano il diritto. Quest'ultimo è acquistabile come ogni merce in circolazione. Una volta che lo si sia fatto proprio, può essere manipolato a piacere.

A fronte della sua base sociale, Berlusconi fa quasi la figura dell'idealista. Nella sua pochezza intellettuale (di cui sono spia gli incidenti grotteschi di cui si rende protagonista a ripetizione), forse crede davvero nella sua missione salvifica; forse pensa sul serio di essere alla testa di una crociata contro un comunismo di cui lui solo scorge traccia. I suoi seguaci del comunismo se ne fottono, così come dell'ideologia in generale. A loro basta un afflusso di denaro liberato da ogni ostacolo. Votano Berlusconi per questo, non per altro; meno che mai perché siano vittime di una manipolazione televisiva.

È vero che Berlusconi, per governare, deve trascinarsi dietro alleati xenofobi, post-fascisti e cattolici integralisti. Non va confuso con questi residuati : per lui sono più che altro uno scotto da pagare al sistema maggioritario cui deve le sue fortune. $\grave{E}$ anche vero che, in politica estera, segue pedissequamente gli Usa. Nemmeno qui ci si deve lasciare fuorviare. Berlusconi non ha alcuna politica estera. Gli incontri internazionali sono per lui ritrovi tra amiconi, dove va per raccontare barzellette o dare consigli ispirati al più generico buonsenso. Se ha partecipato all'invasione 
impact bien délimité, s'il ne venait s'ajouter à une composition sociale profondément altérée. La vulgarité des programmes des chaînes de télévision appartenant à Berlusconi (et aussi, maintenant, des chaînes publiques qui sont entre ses mains) est indiscutable, mais elle trouve son pendant dans la vulgarité culturelle des classes moyennes qui ont atteint leur "conscience de classe.»

Le mot d'ordre «enrichissez-vous!» ne pouvait que créer des légions de parvenus, hédonistes dans le privé et dénués, au niveau social, de quelque éthique que ce soit - exactement comme ce qui s'était passé en Angleterre pendant la période thatchérienne.

Par rapport aux conservateurs traditionnels, ce qui leur manque, ce n'est pas seulement la dignité aristocratique, mais aussi l'ossature morale typique du monde de la production, même quand on le considère du point de vue patronal. Les vieilles vertus capitalistes de l'épargne, de la modération, du réinvestissement sage et prudent ont été balayées.

Du reste, quel rapport direct avec la production peuvent bien avoir ces ribambelles d'avocats, de notaires, d'assureurs, d'opérateurs dans les domaines de l'informatique, de la communication et du spectacle, d'entrepreneurs immobiliers, de boursicoteurs rompus au jeu de faire de l'argent avec de l'argent? Quelle éthique peut bien naître de tout cela? Et s'il s'agit de cadres ou de managers liés à l'univers de la production, la possibilité de tirer aisément avantage de la main-d'œuvre à bas prix des pays de l'Est, ou celle de délocaliser leur petite entreprise dans l'une ou l'autre des régions pauvres de la planète que la mondialisation rend accessibles, ouvrent des perspectives qui suffisent à leur faire oublier la baliverne libérale d'antan, selon laquelle la richesse d'une minorité peut se traduire en richesse sociale, par une cascade de mécanismes intrinsèques.

Ces gens-là ne sont pas des fascistes: cela leur demanderait trop. Ce sont simplement des cyniques. Ils ne cherchent pas d'alibis à ce qu'ils font: c'est le plus fort et le plus chanceux qui gagne, telle est la seule règle. Que ceux qui perdent se débrouillent, la société n'est pas faite pour eux. Et s'il y a des lois inspirées par des conceptions moralistes obsolètes, il n'y a qu'à les ignorer, ou les gommer. Ce sont les rapports de force qui fondent le droit. Lequel peut être acheté, comme toute marchandise en circulation. Une fois qu'on s'en est emparé, on peut le manipuler à loisir.

Si on le compare à sa base sociale, Berlusconi fait pratiquement figure d'idéaliste. Ses moyens intellectuels (que permettent d'apprécier les incidents grotesques dont il est, de façon récurrente, le protagoniste) font qu'il croit peut-être vraiment en sa mission de sauveur; peut-être pense-til pour de bon qu'il est à la tête d'une croisade contre le communisme, 
dell'Iraq, lo ha fatto solo quando ha pensato che la partita fosse chiusa, con lo scrupolo di tenere i soldati italiani bene al caldo.

Berlusconi non è quasi nulla di ciò che gli si attribuisce. Domandare a lui cosa pensa del revisionismo storico, della resistenza, dell'olocausto, è come porre le stesse domande a una delle ochette candidate al titolo di Miss Italia. Con l'unica differenza che, invece di un farfugliamento, si otterranno battute da Bar Sport.

Berlusconi è una cosa sola: economia. Liberismo estremizzato, smantellamento delle forme di coalizione dei lavoratori, fluidità totale dei mercati e della manodopera, ridimensionamento del Welfare, contrazione dell'imposizione fiscale diretta a favore di quella indiretta, fine dei vincoli (da quelli ambientali alle normative antimafia) per le imprese. Questo è Berlusconi, nella sua essenza.

Poi, certo, a tutto ciò il Cavaliere aggiunge il gusto antico degli italiani per la commedia dell'arte, l'irruenza del tifoso da stadio, la compiacenza strumentale verso quella malattia nazionale dell'Italia che è il cinismo. Ciò non toglie che egli sia, principalmente, il rappresentante nello stivale del lascito di Margaret Thatcher e Ronald Reagan, attraverso la mediazione tutta locale del suo maestro Bettino Craxi.

Ciò fa di Berlusconi un problema europeo: ovunque, magari in forme meno pittoresche, sono all'opera forze che professano la medesima ideologia, si chiamino socialdemocratiche o conservatrici. E ne fa un problema per l'opposizione italiana, nelle sue componenti maggioritarie: finché queste ultime faranno propri i capisaldi del programma del Cavaliere, sebbene in forma più blanda e più rispettosa dei vincoli costituzionali, non si vede quale alternativa concreta possano rappresentare. 
dont il est le seul à apercevoir encore la moindre trace. Ses disciples se foutent du communisme, comme de l'idéologie en général. Ce qu'ils veulent, c'est juste la possibilité pour l'argent d'affluer librement. S'ils votent pour Berlusconi, c'est pour cette raison, et pour aucune autre: et certainement pas, en tout cas, parce qu'ils seraient les victimes d'une manipulation télévisuelle.

Il est vrai que pour gouverner, Berlusconi doit accepter d'avoir sur le dos des alliés xénophobes, post-fascistes et catholiques intégristes. Mais il ne faut pas le confondre avec ces scories: à ses yeux, c'est là tout au plus l'écot qu'il doit payer au système majoritaire auquel il doit ses succès. Il est également vrai que, quant à la politique extérieure, il suit servilement les États-Unis. Mais là non plus, il ne faut pas se laisser abuser. Berlusconi n'a aucune politique extérieure. Les rencontres internationales sont pour lui des retrouvailles entre bons copains, où il va pour raconter des histoires drôles ou donner des conseils inspirés par un bon sens des plus banals. S’il a participé à l'invasion de l'Irak, c'est uniquement lorsqu'il a pensé que la partie était jouée, en veillant scrupuleusement à garder les soldats italiens bien au chaud.

Berlusconi n'est à peu près rien de ce qu'on imagine. Lui demander ce qu'il pense du révisionnisme historique, de la résistance, de l'holocauste, revient à poser les mêmes questions à l'une des petites dindes qui concourent pour le titre de Miss Italie. À cette seule différence qu'au lieu de réponses bredouillées, on obtiendra des répliques dignes du café des sports.

Berlusconi est une chose et une seule: économie. Libéralisme poussé à l'extrême, démantèlement des formes de coalition des travailleurs, fluidité totale des marchés et de la main-d'œuvre, réduction des acquis sociaux, abaissement de l'impôt direct au profit de l'impôt indirect, suppression des contraintes (qu'elles concernent l'environnement ou les dispositions anti-mafia) qui pèsent sur les entreprises. Voilà Berlusconi, tel qu'en son essence.

Ensuite, bien sûr, le «Cavaliere" assaisonne tout cela à sa façon : le goût que les Italiens ont de longue date pour la commedia dell'arte, l'élan impétueux du supporter, une complaisance calculée envers cette maladie nationale dont souffre l'Italie qu'est le cynisme. Cela n'ôte rien au fait qu'il est, avant toute chose, le représentant pour la péninsule de Margaret Thatcher et de Ronald Reagan, par l'entremise bien de chez nous de son maitre Bettino Craxi. 
Cela fait de Berlusconi un problème européen : partout, fût-ce sous des formes moins pittoresques, sont à l'œuvre des forces qui professent la même idéologie, qu'elles s'appellent socio-démocrates ou conservatrices. Et cela fait aussi de lui un problème pour l'opposition italienne, du moins pour ses composantes majoritaires: tant que la gauche fera siens les préceptes de fond du programme du Cavaliere, même si c'est en les adoucissant et en respectant davantage les règles constitutionnelles, on ne voit pas quelle alternative concrète elle pourrait représenter. 